This item was submitted to Loughborough's Research Repository by the author.

Items in Figshare are protected by copyright, with all rights reserved, unless otherwise indicated.

\title{
Terminology for analytical capillary electromigration techniques (IUPAC Recommendations 2003)
}

PLEASE CITE THE PUBLISHED VERSION

PUBLISHER

(C) IUPAC

VERSION

VoR (Version of Record)

LICENCE

CC BY-NC-ND 4.0

\section{REPOSITORY RECORD}

Riekkola, Marja-Liisa, Jan Ake Jonsson, and Roger M. Smith. 2019. "Terminology for Analytical Capillary Electromigration Techniques (IUPAC Recommendations 2003)”. figshare. https://hdl.handle.net/2134/4255. 
This item was submitted to Loughborough's Institutional Repository (https://dspace.lboro.ac.uk/) by the author and is made available under the following Creative Commons Licence conditions.

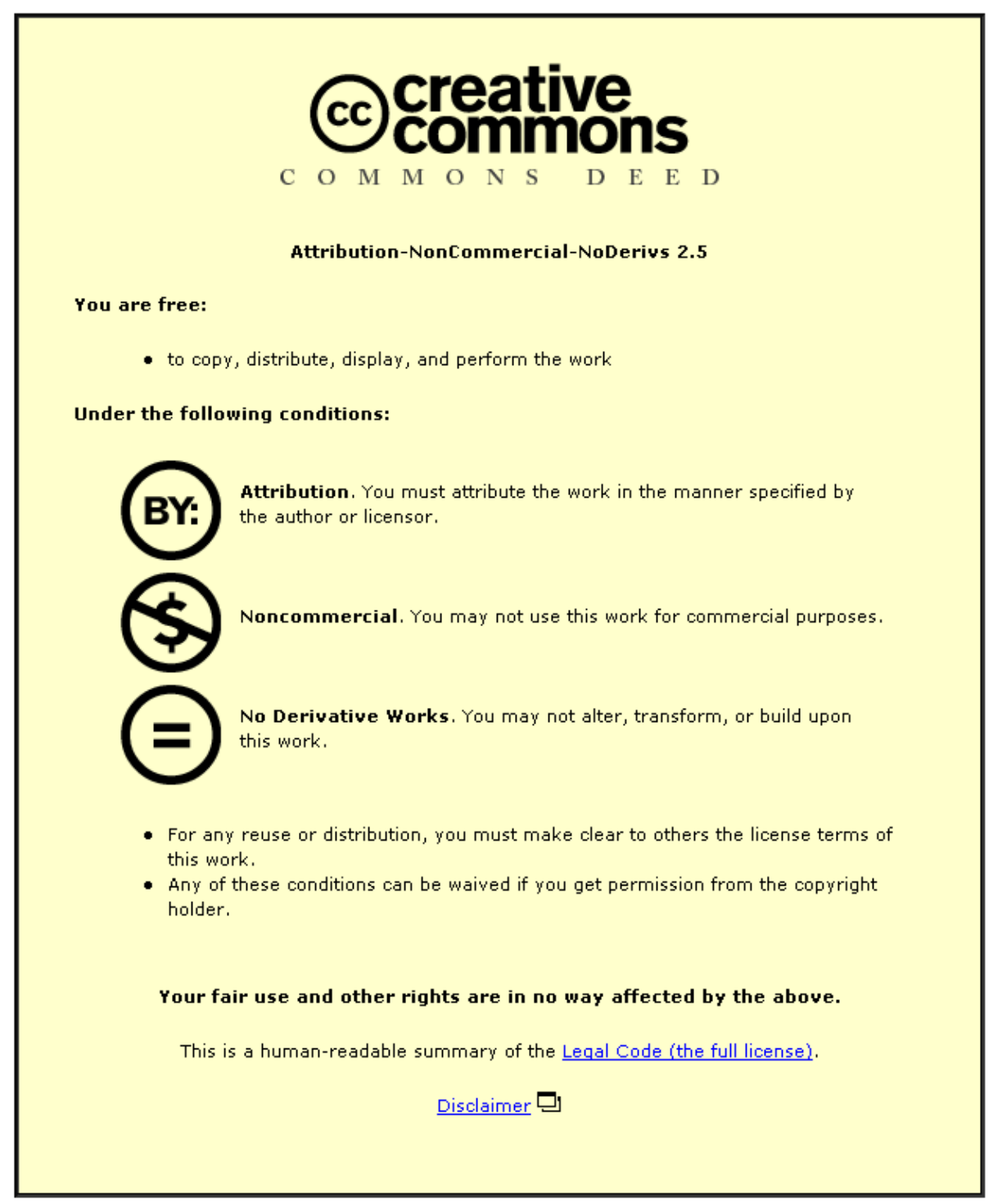

For the full text of this licence, please go to: http://creativecommons.org/licenses/by-nc-nd/2.5/ 
Pure Appl. Chem., Vol. 76, No. 2, pp. 443-451, 2004.

(C) 2004 IUPAC

INTERNATIONAL UNION OF PURE AND APPLIED CHEMISTRY

ANALYTICAL CHEMISTRY DIVISION*

\title{
TERMINOLOGY FOR ANALYTICAL CAPILLARY ELECTROMIGRATION TECHNIQUES
}

(IUPAC Recommendations 2003)

\author{
Prepared for publication by \\ MARJA-LIISA RIEKKOLA ${ }^{1}$, JAN $̊$ KE JÖNSSON ${ }^{2, \ddagger}$, \\ AND ROGER M. SMITH ${ }^{3}$ \\ ${ }^{1}$ Laboratory of Analytical Chemistry, Department of Chemistry, P.O. Box 55, FIN-00014 University of \\ Helsinki, Finland; ${ }^{2}$ Department of Analytical Chemistry, Lund University, P.O. Box 124, \\ SE-221 00 Lund, Sweden; ${ }^{3}$ Department of Chemistry, Loughborough University, \\ Loughborough, Leics. LE11 3TU, UK
}

\begin{abstract}
*Membership of the Analytical Chemistry Division during the final preparation of this report (2002-2003) was as follows:

President: D. Moore (USA); Titular Members: F. Ingman (Sweden); K. J. Powell (New Zealand); R. Lobinski (France); G. G. Gauglitz (Germany); V. P. Kolotov (Russia); K. Matsumoto (Japan); R. M. Smith (UK); Y. Umezawa (Japan); Y. Vlasov (Russia); Associate Members: A. Fajgelj (Slovenia); H. Gamsjäger (Austria); D. B. Hibbert (Australia); W. Kutner (Poland); K. Wang (China); National Representatives: E. A. G. Zagatto (Brazil); M.-L. Riekkola (Finland); H. Kim (Korea); A. Sanz-Medel (Spain); T. Ast (Yugoslavia).
\end{abstract}

"Corresponding author

Republication or reproduction of this report or its storage and/or dissemination by electronic means is permitted without the need for formal IUPAC permission on condition that an acknowledgment, with full reference to the source, along with use of the copyright symbol $\odot$, the name IUPAC, and the year of publication, are prominently visible. Publication of a translation into another language is subject to the additional condition of prior approval from the relevant IUPAC National Adhering Organization. 


\title{
Terminology for analytical capillary electromigration techniques
}

\section{(IUPAC Recommendations 2003)}

\begin{abstract}
This paper presents terms and definitions for capillary electromigration techniques for separation, qualitative and quantitative analysis and physico-chemical characterization. Names and descriptions for such techniques (e.g., capillary electrophoresis and capillary electrochromatography) as well as terms for the phenomenon of electroosmotic flow are included.
\end{abstract}

\section{INTRODUCTION}

Capillary electromigration techniques are becoming increasingly popular and important in chemical analysis, especially in the bioanalytical field. Some of the related terminology can be found in a paper on the terminology of electrophoresis in clinical chemistry [1], but in many cases these are not applicable to capillary techniques and does not fully take into account existing IUPAC definitions [2]. In 1994 Knox published a paper on the terminology of electroseparation techniques [3], but this paper was not harmonized with the IUPAC nomenclature of chromatography [4]. The current paper discusses and defines the relevant terms needed in current practice, including names of the various techniques using electromigration principles. It should be noted that a number of borderline cases exist with respect to the naming of particular techniques.

The separations in capillary electromigration techniques are achieved in narrow capillaries by employing a high electric field strength. These techniques include capillary electrophoretic techniques and electrically driven capillary chromatographic techniques, based on different separation principles. In some cases, these principles overlap. Capillary electromigration techniques have proved to be highly effective for the separation of small organic and inorganic ions, pharmaceuticals, explosives, dyes, polymers, proteins and peptides, DNA and RNA, cells, particles, etc. [5-10]

The presence of electroosmotic flow may contribute to separation even though it is not always needed (e.g., in isoelectric focusing), or may even be completely undesired. The electroosmotic flow originates from the influence of an electric field on the charge in the diffuse part of the double layer at the inner surface of the capillary. As an example, for a fused silica capillary in contact with a solution of $\mathrm{pH}$ above 2, the surface silanol groups are deprotonated, and constitute a negative charge on the capillary wall. These groups give rise to an ion atmosphere, or diffuse layer, next to the wall, in which the population of positively charged counterions predominates. Application of an electric field along the capillary causes the ions in the positively charged diffuse layer to move towards the cathode, which has excess electrons, and therefore appears negatively charged to the cations. At distances greater than that of the surface of shear, where the electroosmotic velocity is zero, the velocity increases rapidly until, at distances greater than the characteristic thickness of the diffuse layer (the Debye length) the velocity becomes essentially constant. For capillary electromigration experiments, the thickness of the diffuse layer is very much smaller than the radius of the capillary, so the electroosmotic flow appears to have a flat profile (so-called plug flow). As a consequence, electroosmotic flow allows high separation efficiencies compared to pressure-driven flow, where the velocity profile is parabolic.

Extremely high separation efficiency, short analysis times, the need for only a small amount of sample, and the low cost of the analysis are advantages available from these techniques. In addition, automated commercial instruments for capillary electromigration techniques allow repeatable and reliable operation in routine analyses. 
Separation capillaries with internal diameters of 20-100 $\mu \mathrm{m}$ and lengths of $20-100 \mathrm{~cm}$ are typically used. In the separation of small molecules, uncoated fused silica capillaries are most commonly employed. To prevent surface adsorption of the analytes or matrix components, and to eliminate or control the electroosmotic flow, surface coating or chemical modification of the silanol groups may be necessary. Neutral and charged coatings have been successfully used in a variety of applications.

Sample injection into the capillary can be performed hydrodynamically by creating a pressure difference between the buffer and the sample solution reservoirs, while dipping the appropriate end of the separation capillary into a sample solution. Using electrokinetic injection, a small injection voltage is applied for a defined time, while the appropriate end of the separation capillary is inserted in the sample reservoir, and the other end is in a buffer reservoir.

Ideally, the zone dispersion effects during the separation are due only to longitudinal diffusion. However, electrodispersion (caused by differences in mobilities between buffer and sample), as well as temperature gradients inside the capillary may cause additional band broadening effects. The sample introduction technique, sample overloading, the type of sample matrix and adsorption phenomena on the capillary walls may become additional sources of zone dispersion. Temperature control of the separation capillary, the choice of an appropriate buffer medium, the surface treatment technique, the sample type and the way the sample is introduced into the capillary may all be crucial to the success of a particular separation.

Additives, such as organic solvents, cyclodextrins or polymers, can be used to control the migration and peak shapes of solutes. Cyclodextrins can also be employed as a part of wall coatings, rigid gels, and micellar dispersed phases. Capillaries filled with cross-linked e.g., polyacrylamide gels can be used for the size-based separation of large biomolecules. The incorporation of different chiral selector molecules into such a gel system has been successful for the chiral separations of small molecules. Solutions of entangled linear polymers are frequently used in the separation of biomolecules.

In capillary electrochromatography [11,12], the separations are carried out in capillary columns containing particulate packings as used in liquid chromatography or monolithic materials. Coated open tubular capillaries, similar to gas chromatographic capillary columns can also be employed.

In the future, microchannels on a chip-like structure are likely to be exploited more frequently in capillary electromigration techniques after further development of nanotechnology.

\section{CAPILLARY ELECTROMIGRATION TECHNIQUES}

\section{capillary electrophoresis (CE)}

A separation technique carried out in capillaries based solely on the differences in the electrophoretic mobilities of charged species (analytes) either in aqueous or non-aqueous background electrolyte solutions. These can contain additives, which can interact with the analytes and alter their electrophoretic mobility.

Notes:

1. This technique is also known as capillary zone electrophoresis (CZE).

2. The use of the term capillary electrophoresis (CE) as a collective term for all capillary electromigration techniques is not recommended as some of these techniques involve other separation mechanisms than electrophoresis.

\section{capillary affinity electrophoresis (CAE)}

An electrophoretic separation technique, which takes place in a capillary, with the background electrolyte containing substances capable of specific, often biospecific, interactions with the analytes.

Note: Also called affinity capillary electrophoresis (ACE). 


\section{capillary sieving electrophoresis (CSE)}

An electrophoretic separation technique, which takes place in a capillary, containing a sieving medium (e.g., an entangled polymer network) in the background electrolyte. The separation is based on differences in size and shape of the charged analytes.

\section{capillary gel electrophoresis (CGE)}

A special case of capillary sieving electrophoresis when the capillary is filled with a cross-linked gel.

\section{capillary isoelectric focusing (CIEF)}

An electrophoretic technique for the separation of amphoteric analytes according to their isoelectric points by the application of an electric field along a $\mathrm{pH}$ gradient formed in a capillary.

\section{capillary isotachophoresis (CITP)}

An electrophoretic separation technique in a discontinuous buffer system in which the analytes migrate according to their electrophoretic mobilities, forming a chain of adjacent zones moving with equal velocity between two solutions, leading and terminating electrolytes, bracketing the mobility range of the analytes.

\section{electrokinetic chromatography (EKC)}

A separation technique based on a combination of electrophoresis and interactions of the analytes with additives (e.g., surfactants), which form a dispersed phase moving at a different velocity. In order to achieve separation either the analytes or this secondary phase should be charged.

Notes:

1. This is not totally compatible with the definition of chromatography.

2. The same technique is also called electrokinetic capillary chromatography (ECC).

\section{micellar electrokinetic chromatography (MEKC)}

A special case of electrokinetic chromatography, in which the secondary phase is a micellar dispersed phase in the capillary. (MECC).

Note: The same technique is also called micellar electrokinetic capillary chromatography

\section{microemulsion electrokinetic chromatography (MEEKC)}

A special case of electrokinetic chromatography, where a microemulsion is employed as the dispersed phase.

Note: The same technique is also called microemulsion electrokinetic capillary chromatography (MEECC).

\section{capillary electrochromatography (CEC)}

A special case of capillary liquid chromatography, where the movement of the mobile phase through a capillary, filled, packed or coated with a stationary phase, is achieved by electroosmotic flow (which may be assisted by pressure). The retention time is determined by a combination of electrophoretic migration and chromatographic retention. 


\section{TERMS RELATED TO CAPILLARY ELECTROMIGRATION TECHNIQUES}

\section{electroosmosis}

Motion of a liquid through a membrane (or plug or capillary) as a consequence of the application of an electric field across the membrane.

Notes:

1. The linear velocity of flow divided by field strength is called the electroosmotic mobility $(u$ or $\left.\mu_{\mathrm{eo}}\right)$.

2. The spelling of electro-osmosis with two o's (with or without a hyphen) is preferable to electrosmosis with one $\mathrm{o}$ and to the older term electroendosmosis.

Corrected from [2].

\section{electroosmotic volume flow}

The volume flow divided by field strength through the whole plug or capillary.

Corrected from [2].

electroosmotic hold-up time (in capillary electromigration), $t_{\mathrm{eo}}$

Time required for a liquid in a capillary to move due to electroosmosis through the effective length of the capillary, $L_{\text {eff. }}$. This time is usually measured as the migration time of a neutral compound, called an electroosmotic flow marker, which is assumed to have an electroosmotic mobility that is negligible compared to that of the analyte.

electroosmotic velocity, $v_{\mathrm{eo}}$

See electroosmosis [2].

Note: In capillary electromigration, this velocity is positive, by convention, in the direction from the sample introduction end of the capillary to the detection end, otherwise negative. The electroosmotic velocity can be calculated as $v_{\mathrm{eo}}=L_{\mathrm{eff}} / t_{\mathrm{eo}}$, the effective length of the capillary divided by the electroosmotic hold-up time.

electric field (strength), $E$

Force acting on a charge divided by the charge [2].

Note: In capillary electromigration, this is taken as the axial component of the field, that is, the difference between the applied electric potential at the sample introduction end of the capillary and that at the detection end, divided by the total length of the capillary, $L_{\text {tot }}$. There is also a radial electric field arising from the electrical double layer, which is involved in electroosmosis. The total length of the capillary is typically larger than the effective length of the capillary, $L_{\mathrm{eff}}$. See migration time.

electroosmotic mobility (in capillary electromigration), $u$ or $\mu_{\mathrm{eo}}$ electroosmotic velocity divided by electric field (strength).

$$
\mu_{\mathrm{eo}}=\frac{v_{\mathrm{eo}}}{\boldsymbol{E}}
$$

electrokinetic potential, $\zeta$

Potential drop across the mobile part of the double layer, that is responsible for electrokinetic phenomena. $\zeta$ is positive if the potential increases from the bulk of the liquid phase towards the interface. In calculating the electrokinetic potential from electrokinetic phenomena it is often assumed that the liquid adhering to the solid wall and the mobile liquid are separated by a sharp shear plane. As long as there is no reliable information on the values of the permittivity and the viscosity in the electrical double layer close to the interface, the calculation of the electrokinetic potential from electrokinetic experiments is open to criticism. It is therefore essential to indicate in all cases which equations have been 
used in the calculation of $\zeta$. It can be shown, however, that for the same assumptions about the permittivity and viscosity all electrokinetic phenomena must give the same value for the electrokinetic potential [2].

Notes:

1. If the conditions referred to are followed, the electrokinetic potential can be related to the electroosmotic mobility, $\mu_{\mathrm{eo}}$, by the Smoluchowski equation:

$$
\mu_{\mathrm{eo}}=-\frac{\varepsilon_{\mathrm{r}} \varepsilon_{0} \zeta}{\eta}
$$

where $\varepsilon_{\mathrm{r}}$ is the relative permittivity of the solution (usually taken as that of the pure solvent), $\varepsilon_{0}$ is the permittivity of vacuum, and $\eta$ is the dynamic viscosity of the solution, where the ratio of permittivity to viscosity is assumed to be independent of the electric field of the double layer.

2. Electrokinetic potential is often called $\zeta$-potential or zeta potential.

\section{migration time, $t_{\mathrm{m}}$}

The time required for the analyte to move through the effective length of the capillary, $L_{\text {eff }}$, that is, from the sample introduction point to the detection point.

rate of migration (in electrophoresis), $v$

The distance of migration divided by time, sometimes called velocity of migration. The symbol $v$ is also used for velocity [2].

\section{electrophoretic velocity, $v_{\mathrm{ep}}$}

The velocity of a charged analyte under the influence of an electric field relative to the background electrolyte. Also called rate of migration (in electrophoresis).

\section{electrophoretic mobility, $\mu$ or $\mu_{\mathrm{ep}}$}

The observed rate of migration (in electrophoresis), $v$, (or electrophoretic velocity, $v_{\mathrm{ep}}$ ) divided by the magnitude of the electric field strength, $\boldsymbol{E}$, in a given medium.

Notes:

1. Mobilities are sometimes expressed with a negative sign, because migration of the solutes or particles generally occurs in a direction opposite to the direction of the electric field (which is taken as a reference for that direction).

2. In a solid support medium, only apparent values can be determined. Changed from [2].

3. The electrophoretic mobility of an ion is given by:

$$
\mu_{\mathrm{ep}}=\frac{z e}{f}
$$

In this equation, $f$ is the friction coefficient, $z$ is the number of elementary charges on the ion and $e$ is the elementary charge. The friction coefficient increases with the size of the ion and with the viscosity of the supporting (background) electrolyte. For rigid spherical ions, of radius $r$, moving in a continuum of viscosity $\eta$, the frictional coefficient may be estimated using Stokes law as:

$$
f=6 \pi \eta r
$$

Friction coefficients for particles of other shapes, such as oblate or prolate ellipsoids, may be estimated using more complex equations. 
total velocity of the analyte (in capillary electrophoresis), $v_{\text {tot }}$

The sum of the electrophoretic velocity, $v_{\mathrm{ep}}$, of an ion and the electroosmotic velocity, $v_{\mathrm{eo}}$.

$$
v_{\mathrm{tot}}=v_{\mathrm{ep}}+v_{\mathrm{eo}}
$$

This quantity can be measured experimentally as the effective length of the capillary divided by the migration time $\left(L_{\mathrm{eff}} / t_{\mathrm{m}}\right)$.

Notes:

1. Depending on the signs and relative magnitudes of these velocities, the total velocity of an analyte can have either the same or the opposite direction to the electroosmotic velocity.

2 . The total velocity is the velocity of the ion measured as a displacement relative to the capillary wall divided by time.

migration time of micelles (in micellar electrokinetic chromatography), $t_{\mathrm{mc}}$

Experimentally determined as the migration time of a compound that is completely retained in the micellar phase.

mass distribution ratio (in micellar electrokinetic chromatography), $k_{\mathrm{MEKC}}$

Defined as:

$$
k_{\mathrm{MEKC}}=\frac{n_{\mathrm{mc}}}{n_{\mathrm{aq}}}=K \cdot \frac{V_{m c}}{V_{a q}}
$$

where $n_{\mathrm{mc}}$ and $n_{\mathrm{aq}}$ are the chemical amounts of the analyte in the micellar and aqueous phases, respectively, $K$ is the distribution constant and $V_{\mathrm{mc}}$ and $V_{\mathrm{aq}}$ are the corresponding volumes of the phases.

Notes: tion times:

1. In the case of an electrically neutral analyte, $k_{\mathrm{MEKC}}$ can be calculated directly from the migra-

$$
k_{\mathrm{MEKC}}=\frac{\left(t_{\mathrm{m}}-t_{\mathrm{eo}}\right)}{t_{\mathrm{eo}}\left(1-t_{\mathrm{m}} / t_{\mathrm{mc}}\right)}
$$

2. $k_{\mathrm{MEKC}}$ should not be confused with the retention factor (in column chromatography) $k$. However, $k_{\mathrm{MEKC}}$ is analogous to the mass distribution ratio (in chromatography).

mass distribution ratio (in microemulsion electrokinetic chromatography), $k_{\mathrm{MEEKC}}$

Defined analogously to mass distribution ratio (in micellar electrokinetic chromatography), $k_{\mathrm{MEKC}}$ by replacing terms for micelles with corresponding terms for microemulsion. 


\section{LIST OF ACRONYMS}

ACE

CAE

$\mathrm{CE}$

CEC

CGE

CIEF

CITP

CZE

CSE

EKC

MECC

MEECC

MEEKC

MEKC

\section{SYMBOLS}

\section{$e$}

E

$f$

K

$k_{\text {MEEKC }}$

$k_{\mathrm{MEKC}}$

$L_{\text {eff }}$

$L_{\text {tot }}$

$n_{\mathrm{aq}}$

$n_{\mathrm{mc}}$

$r$

$t_{\text {eo }}$

$t_{\mathrm{m}}$

$t_{\mathrm{mc}}$

$V_{\text {aq }}$

$V_{\mathrm{mc}}$

$z$

$\varepsilon_{\mathrm{r}}$

$\varepsilon_{0}$

$\zeta$

$\eta$

$\mu$

$\mu_{\text {eo }}$

$\mu_{\text {ep }}$

$v$

$v_{\text {eo }}$

$v_{\text {ep }}$

$v_{\text {tot }}$ affinity capillary electrophoresis

capillary affinity electrophoresis

capillary electrophoresis

capillary electrochromatography

capillary gel electrophoresis

capillary isoelectric focusing

capillary isotachophoresis

capillary zone electrophoresis

capillary sieving electrophoresis

electrokinetic chromatography

micellar electrokinetic capillary chromatography

microemulsion electrokinetic capillary chromatography

microemulsion electrokinetic chromatography

micellar electrokinetic chromatography

elementary charge

electric field (strength)

friction coefficient

distribution constant

mass distribution ratio in microemulsion electrokinetic chromatography

mass distribution ratio in micellar electrokinetic chromatography

effective length of the capillary

total length of the capillary

chemical amount of the analyte in the aqueous phase

chemical amount of the analyte in the micellar phase

Stokes radius

electroosmotic hold-up time

migration time of the analyte

migration time of micelles

volume of the aqueous phase

volume of the micellar phase

number of elementary charges

permittivity of the solution

permittivity of vacuum

zeta potential or electrokinetic potential

dynamic viscosity

electrophoretic mobility

electroosmotic mobility

electrophoretic mobility

rate of migration

electroosmotic flow velocity

electrophoretic velocity

total velocity of the analyte 


\section{REFERENCES}

1. G. Férard. "Quantities and units for electrophoresis in the clinical laboratory", Pure Appl. Chem. 66, 891-896 (1994).

2. A. D. McNaught and A. Wilkinson. Compendium of Chemical Terminology (The Gold Book), $2^{\text {nd }}$ ed., Blackwell Science, Oxford (1997).

3. J. H. Knox. "Terminology and nomenclature in capillary electroseparation systems", $J$. Chromatogr. A 680, 3-13 (1994).

4. L. E. Ettre. "Nomenclature for chromatography", Pure Appl. Chem. 65, 819-872 (1993).

5. F. Foret, L. Krivankova, P. Bocek. Capillary Zone Electrophoresis, VCH, Weinheim (1993).

6. H. Engelhardt, W. Beck, T. Schmitt. Capillary Electrophoresis. Methods and Potentials, Vieweg, Wiesbaden (1996).

7. P. Camillari. Capillary Electrophoresis, $2^{\text {nd }}$ ed., CRC Press, Boca Raton, FL (1998).

8. D. E. Baker. Capillary Electrophoresis, John Wiley, New York (1995).

9. M. G. Khaledi. High-Performance Capillary Electrophoresis, John Wiley, New York (1998).

10. W. Kok. "Capillary electrophoresis: Instrumentation and operation", Chromatographia supplement 51 (2000).

11. Z. Deyl and F. Svec. Capillary Electrochromatography, Elsevier Science, Amsterdam (2001).

12. K. Bartle and P. Myers. Capillary Electrochromatography, Royal Society of Chemistry, Cambridge (2001). 